\title{
FREE PRODUCTS OF TOPOLOGICAL GROUPS WITH CENTRAL AMALGAMATION. II
}

\author{
BY
}

\author{
M. S. KHAN AND SIDNEY A. MORRIS
}

\begin{abstract}
In Free products of topological groups with central amalgamation. I, we introduced the notion of amalgamated free product of topological groups and showed that if $A$ is a common central closed subgroup of Hausdorff topological groups $G$ and $H$, then the amalgamated free product $G \amalg_{A} H$ exists and is Hausdorff. In this paper, we give an alternative much shorter (but less informative) proof of this result. We then proceed to describe the properties of $G \amalg_{A} H$. In particular, we find necessary and sufficient conditions for $G \amalg_{A} H$ to be a locally compact Hausdorff group, a complete metric group, and a maximally almost periodic group. Properties such as being a Baire space and connectedness are also investigated. In the case that $G, H$ and $A$ are $k_{\omega}$-groups, the topology of $G \amalg_{A} H$ is fully described. A consequence of this description is that for $k_{\omega}$-groups $G \amalg_{A} H$ is homeomorphic to $\left(G \times{ }_{A} H\right) \times$ $F(G / A \wedge H / A)$, where $G \times{ }_{A} H$ is the direct product of $G$ and $H$ with $A$ amalgamated, and $F(G / A \wedge H / A)$ is the free topological group on the smash product of $G / A$ and $H / A$.
\end{abstract}

1. Introduction and preliminaries. In $1950 \mathrm{M}$. I. Graev [4] proved that the free product of any two Hausdorff topological groups exists and is Hausdorff.

In [9] we considered amalgamated free products of topological groups, which are defined as follows:

Definition. Let $A$ be a common subgroup of topological groups $G$ and $H$. The topological group $G \amalg_{A} H$ is said to be the free product of the topological groups $G$ and $H$ with amalgamated subgroup $A$ if

(i) $G$ and $H$ are topological subgroups of $G \amalg_{A} H$,

(ii) $G \cup H$ generates $G \amalg_{A} H$ algebraically, and

(iii) every pair $\phi_{1}, \phi_{2}$ of continuous homomorphisms of $G$ and $H$, respectively, into any topological group $D$, which agree on $A$, extend to a continuous homomorphism of $G \amalg_{A} H$ into $D$.

Using a standard categorical argument one can prove the following existence theorem:

THEOREM A. Let $G$ and $H$ be topological groups with a common subgroup A. Let $f_{1}$ : $A \rightarrow G$ and $f_{2}: A \rightarrow H$ be the topological group isomorphisms of $A$ onto subgroups of $G$ and $H$, respectively. Then the amalgamated free product $G \amalg_{A} H$ exists if and only if there is a topological group $E$ and there are continuous homomorphisms $\phi_{1}$ and $\phi_{2}$ of $G$ and $H$, respectively, into $E$ such that $\phi_{1}$ and $\phi_{2}$ are topological group isomorphisms of $G$ and $H$ onto their respective images in $E$ and $\phi_{1} f_{1}=\phi_{2} f_{2}$.

Received by the editors June 12, 1981.

1980 Mathematics Subject Classification. Primary 22A05, 22D99, 20 E06.

(C)1982 American Mathematical Society 0002-9947/81/0000-1021/\$05.00 
Notation. Whenever $A$ is a common subgroup of topological groups $G$ and $H$, we shall denote the embeddings of $A$ in $G$, and $A$ in $H$ by $f_{1}$ and $f_{2}$, respectively.

Remarks. (i) If $A=\{e\}$, the identity element, then $G \amalg_{A} H$ is simply the free product $G \amalg H$ of the topological groups $G$ and $H$. Further, the above theorem implies the existence of $G \amalg H$, since we can put $E=G \times H$.

(ii) If $A$ is a central subgroup of $G$ and $H$, then the above theorem guarantees the existence of $G \amalg_{A} H$ since we can put $E=G \times_{A} H$, the direct product of $G$ and $H$ with $A$ amalgamated.

Definition [8]. A topological group $G \times_{A} H$ is said to be the direct product of its topological subgroups $G$ and $H$, with amalgamated subgroup $A$ if it has the properties

(i) $G \times{ }_{A} H$ is algebraically generated by $G \cup H$,

(ii) $G \cap H=A$,

(iii) $G$ is contained in the centralizer of $H$ in $G \times{ }_{A} H$, and

(iv) if $\phi_{1}$ and $\phi_{2}$ are any continuous homomorphisms of $G$ and $H$, respectively, into any topological group $E$ such that $\phi_{1}\left|A=\phi_{2}\right| A$ and $\phi_{2}(G)$ is contained in the centralizer of $\phi_{2}(H)$ in $E$, then there exists a continuous homomorphism $\Phi$ : $G \times{ }_{A} H \rightarrow E$ such that $\Phi \mid G=\phi_{1}$ and $\Phi \mid H=\phi_{2}$.

In [8] it is shown that given $A$ a common subgroup of topological groups $G$ and $H$, $G \times{ }_{A} H$ exists (and is unique) if and only if $A$ is a central subgroup of both $G$ and $H$.

It is a routine matter to prove the uniqueness of $G \amalg_{A} H$, if it exists. Indeed, one can show that the underlying group structure of $G \amalg_{A} H$ is that of $G *_{A} H$, the (algebraic) free product of $G$ and $H$ with $A$ amalgamated. (The standard references on amalgamated free products of groups are [19 and 11].) Further, the topology of $G \amalg_{A} H$ is the finest group topology on $G *_{A} H$ which will induce the given topologies on $G$ and $H$.

In [9] and this paper we restrict our attention primarily to the case when $A$ is a central subgroup of both $G$ and $H$. So, as remarked above, the existence (and uniqueness) of $G \amalg_{A} H$ is guaranteed. The task which we set ourselves is that of describing the topology of $G \amalg_{A} H$, given that of $G, H$ and $A$.

Of course the first task is to settle the question of Hausdorffness. Indeed, it was shown in [9] that if $A$ is a common closed central subgroup of Hausdorff groups $G$ and $H$, then $G \amalg_{A} H$ is Hausdorff. This result includes that of Graev [5] and indeed our argument is a (nontrivial) extension of his.

In $\S 2$ of this paper we give an alternative proof of the Hausdorffness of $G \amalg_{A} H$. This argument is much simpler than that presented in [9], but in no way replaces the earlier proof as it provides less information.

In $\S 4$ we completely describe the topology of $G \amalg_{A} H$ in the case of $k_{\omega}$-groups. A nice consequence of this description is the fact that $G \amalg_{A} H$ is homeomorphic to $\left(G \times_{A} H\right) \times F(G / A \wedge H / A)$, where $G \times_{A} H$ is the amalgamated direct product of $G$ and $H$, and $F(G / A \wedge H / A)$ is the (Graev) free topological group on the smash product $G / A \wedge H / A$. (For a discussion of free topological groups see [14, 4, 12 and 22].)

In $\$ 3$ we consider non- $k_{\omega}$-groups where we are unable to fully describe the topology of $G \amalg_{A} H$. Nevertheless, we find necessary and sufficient conditions for 
$G \amalg_{A} H$ to be a locally compact Hausdorff group, a complete metric group, and a maximally almost periodic group. Other properties such as being a Baire space are also investigated.

Notation. We denote the algebraic free product of two groups $G$ and $H$ by $G * H$. Also if $G$ and $H$ are subgroups of a group $E$, then we use the symbol [ $G, H$ ] to denote the set $\left\{g^{-1} h^{-1} g h: g \in G\right.$ and $\left.h \in H\right\}$.

For completeness we include the following definition from [9]: Given pseudometrics $\rho_{G}$ and $\rho_{H}$, respectively, we define a pseudometric $\rho_{X}$ on the set $[G, H] \subset G *_{A} H$ by

$$
\rho_{X}\left(\left[g_{1}, h_{1}\right],\left[g_{2}, h_{2}\right]\right)=\inf _{a_{1}, a_{2} \in A}\left(\operatorname { m i n } \left\{\min \left[\rho_{G}\left(g_{1}, a_{1}\right) ; \rho_{H}\left(h_{1}, a_{1}\right)\right]\right.\right.
$$

$$
\begin{aligned}
+\min [ & \left.\rho_{G}\left(g_{2}, a_{2}\right) ; \rho_{H}\left(h_{2}, a_{2}\right)\right] ; \\
& \left.\left.\rho_{G}\left(g_{1} a_{1}, g_{2}\right)+\rho_{H}\left(h_{1} a_{2}, h_{2}\right)\right\}\right)
\end{aligned}
$$

where $g_{1}, g_{2} \in G$ and $h_{1}, h_{2} \in H$.

2. An alternative proof of Hausdorffness. In [9] it was proved that if $A$ is a common closed central subgroup of Hausdorff groups $G$ and $H$, then the amalgamated free product $G \amalg_{A} H$ is Hausdorff. The proof was rather long and was based on Graev's proof [5] of the Hausdorffness of the free product $G \amalg H$. Because of the importance of this result we give a different and much shorter proof here, which unlike the other proof may be able to be extended to the case of noncentral amalgamations. This shorter proof depends on knowing that $G \amalg H$ is Hausdorff and does not replace the earlier proof of [9] as it gives less information about the topology of $G \amalg_{A} H$. For example, later we prove that if $G$ and $H$ are Lie groups then $G \amalg_{A} H$ is an NSS-group, using information available in the first proof but not this proof.

The proof of Hausdorffness requires two preliminary results.

Proposition 1. Let $A$ be a common central subgroup of groups $G$ and $H$ and $\Gamma$ the canonical homomorphism of $G * H$ onto $G *_{A} H$. Then an element $u=g h k$, where $g \in G, h \in H$ and $k \in K(G * H)=$ the cartesian subgroup of $G * H$, lies in the kernel of $\Gamma$ if and only if

(i) $g=f_{1}(a)$ and $h=f_{2}\left(a^{-1}\right)$, for some $a \in A$, and

(ii) $k \in \operatorname{ngp}\left\{\left[f_{1}(A), H\right] \cup\left[G, f_{2}(A)\right]\right\}=$ the normal subgroup of $K(G * H)$ generated by $\left[f_{1}(A), H\right] \cup\left[G, f_{2}(A)\right]$.

Proof. If condition (i) is satisfied then $\Gamma(g h)=e$. If condition (ii) is satisfied then $\Gamma(k)=e$. So together they imply that $\Gamma(u)=e$.

Conversely, let $\Lambda$ be the canonical homomorphism of $G *_{A} H$ onto the amalgamated direct product $G \times{ }_{A} H$. Then $\Gamma(u)=e$ implies $\Lambda \Gamma(u)=e$; that is, $g h=e$ in $G \times{ }_{A} H$, which implies that $g=f_{1}(a)$ and $h=f_{2}\left(a^{-1}\right)$, for some $a \in A$. So condition (i) is satisfied. Thus $\Gamma(g h)=e$ and then $\Gamma(g h k)=e$ implies $\Gamma(k)=e$. 
Now we can write $k=k_{1} \cdot k_{2}$, where $k_{1} \in \operatorname{ngp}\left\{\left[f_{1}(A), H\right] \cup\left[G, f_{2}(A)\right]\right\}$ and $k_{2}=\left[g_{1}^{\prime}, h_{1}^{\prime}\right]^{\gamma_{1}}\left[g_{2}^{\prime}, h_{2}^{\prime}\right]^{\gamma_{2}} \cdots\left[g_{s}^{\prime}, h_{s}^{\prime}\right]^{\gamma_{s}}$, where $g_{i}^{\prime} \in G \backslash f_{1}(A)$ and $h_{i}^{\prime} \in H \backslash f_{2}(A)$ for $i=1, \ldots, s$, and each $\gamma_{i}$ is an integer. Clearly $\Gamma\left(k_{1}\right)=e$. So $\Gamma(k)=e$ implies $\Gamma\left(k_{2}\right)=e$. But by Proposition 1 of [9] this implies that $k_{2}=e$. So $k=k_{1}$ and condition (ii) is satisfied.

Notation. In [9] we introduced a topology $\tau$ on $G *_{A} H$. (In the case that $A=\{e\}$, and $G *_{A} H=G * H$, this topology is still denoted by $\tau$ and coincides with the topology Graev constructs in [5].) The topology arises from pseudometrics $\rho_{X}$ defined by equation (1) of [9]. (See $\$ 1$ of this paper.)

LEMMA 1. Let $G$ and $H$ be Hausdorff topological groups. If $S$ is a closed subspace of $G$, then the subset $[S, H]=\left\{s^{-1} h^{-1} s h: s \in S, h \in H\right\}$ of $G * H$ is a closed set in the topology $\tau$.

Proof. It suffices to show that if $[g, h]$ is any element such that $g \in G \backslash S$ and $h \in H \backslash\{e\}$ then there exists a $\rho_{X}$ (of the type mentioned above) such that

$$
\rho_{X}\left([g, h],\left[s, h_{1}\right]\right)>1, \text { for all elements }\left[s, h_{1}\right] \text { of }[S, H] .
$$

As $S$ is closed in $G$, there exists a continuous pseudometric $\rho_{G}$ on $G$ such that $\rho_{G}(g, s)>1$, for all $s \in S$. As $H$ is Hausdorff, there exists a continuous pseudometric $\rho_{H}$ on $H$ such that $\rho_{H}(h, e)>1$. Let $\rho_{X}$, defined by equation (1) of [9] with $A=\{e\}$, be the pseudometric corresponding to $\rho_{G}$ and $\rho_{H}$. Then by definition

$$
\rho_{X}\left([g, h],\left[s, h_{1}\right]\right)>1, \text { for all } s \in S \text { and } h_{1} \in H .
$$

Corollary. Let $G$ and $H$ be Hausdorff topological groups. If $A_{1}$ and $A_{2}$ are closed subgroups of $G$ and $H$ respectively, then the normal subgroup $\operatorname{ngp}\left\{\left[A_{1}, H\right] \cup\left[G, A_{2}\right]\right\}$ of $G * H$ generated by $\left[A_{1}, H\right] \cup\left[G, A_{2}\right]$ is a closed set in the topology $\tau$ and hence also in $G \amalg H$.

Proof. This is an immediate consequence of Lemma 1 and Graev [4, §4D]. (The latter result is that if $Z$ is a closed subspace of the completely regular Hausdorff space $Y$, then $\operatorname{ngp}(Z)$ is a closed subspace of the free group on the set $Y \backslash\{e\}$ when this is topologized in Graev's pseudometric fashion.)

THEOREM 1. Let $A$ be a common closed central subgroup of the Hausdorff topological groups $G$ and $H$. Then $G \amalg_{A} H$ is Hausdorff.

Proof. To prove this it suffices to show that the kernel of the canonical homomorphism $\Gamma: G \amalg H \rightarrow G \amalg_{A} H$ is closed.

By Theorem 2 of [18], $G \amalg H$ is homeomorphic to $G \times H \times K(G \amalg H)$, where $K(G \amalg H)$ is topologized as a subspace of $G \amalg H$. Further by Graev [5] or Khan-Morris [9], this topology on $K(G \amalg H)$ is at least as fine as the topology $\tau$ mentioned above. From Proposition 1, the kernel of $\Gamma$ is the subspace

$$
Q \times \operatorname{ngp}\left\{\left[f_{1}(A), H\right] \cup\left[G, f_{2}(A)\right]\right\}
$$

of $G \times H \times K(G \amalg H)$ where

$$
Q=\left\{\left(f_{1}(a), f_{2}\left(a^{-1}\right)\right): a \in A\right\} .
$$


As $f_{1}(A)$ is closed in $G$ and $f_{2}(A)$ is closed in $H$, the "diagonal" copy of $A$, namely $Q$, is closed in $G \times H$. So to prove that the kernel of $\Gamma$ is closed it suffices to show that $\operatorname{ngp}\left\{\left[f_{1}(A), H\right] \cup\left[G, f_{2}(A)\right]\right\}$ is closed in $K(G \amalg H)$. But this follows immediately from the Corollary to Lemma 1.

3. Local compactness and other properties. In this section we investigate the question: If $G$ and $H$ have some property $P$, under what circumstances does $G \amalg_{A} H$ also have property $P$ ? Some of these questions are trivially answered, for example, connectivity, while others are not. Particularly noteworthy is the fact that $G \amalg_{A} H$ can be nondiscrete and locally compact. (This may prove to be a source of interesting examples or counterexamples but we have not pursued this aspect here.) This should be contrasted with the previously known result for $G \amalg H$, namely that it is locally compact if and only if $G, H$ and $G \amalg H$ are discrete or $G=\{e\}$ or $H=\{e\}$.

The first result is an immediate consequence of the fact that $G \amalg_{A} H$ is algebraically generated by $G \cup H$ and that $G \cap H \neq \varnothing$.

Proposition 2. Let A be a common subgroup (not necessarily closed or central) of topological groups $G$ and $H$. If $G$ and $H$ are connected (respectively, path connected) then $G \amalg_{A} H$ (if it exists) is connected (respectively, path connected).

Remark. The converse of Proposition 2 is false. For example if $\mathbf{Z}_{i} \subset \mathbf{R}_{i}, i=1,2$, denote copies of the discrete group of integers and the additive group of reals with the usual topology, then the amalgamated free product

$$
\left(\mathbf{R}_{1} \times \mathbf{Z}_{2}\right) \mathbf{\amalg}_{\mathbf{Z}_{1} \times \mathbf{Z}_{2}}\left(\mathbf{Z}_{1} \times \mathbf{R}_{2}\right)
$$

is a path connected group (indeed it is topologically isomorphic to $\mathbf{R}_{1} \mathbf{U} \mathbf{R}_{2}$ ) but neither $\mathbf{R}_{1} \times \mathbf{Z}_{2}$ nor $\mathbf{Z}_{1} \times \mathbf{R}_{2}$ is connected.

Observing that the topology of $G \amalg_{A} H$ is the finest group topology on $G *_{A} H$ which induces the given topologies on $G$ and $H$, we obtain the following.

Proposition 3. Let $A$ be a common (not necessarily central) subgroup of topological groups $G$ and $H$. Then $G \amalg_{A} H$ (if it exists) has the discrete topology if and only if $G$ and $H$ have the discrete topology.

THEOREM 2. Let $A$ be a common closed central subgroup of the locally compact Hausdorff groups $G$ and $H$. Then $G \amalg_{A} H$ has no small subgroups if and only if $G$ and $H$ are Lie groups.

Proof. If $G \amalg_{A} H$ has no small subgroups, then its subgroups $G$ and $H$ have no small subgroups. But the solution to Hilbert's fifth problem [7] says that a locally compact Hausdorff group is a Lie group if and only if it has no small subgroups. So $G$ and $H$ are Lie groups.

Conversely, if $G$ and $H$ are Lie groups then the quotient groups $G / A$ and $H / A$ are Lie groups. Let $\rho_{1}$ and $\rho_{2}$ be admissible metrics on $G / A$ and $H / A$, respectively. Define the corresponding continuous pseudometrics $\rho_{G}$ and $\rho_{H}$, on $G$ and $H$, respectively, by $\rho_{G}\left(g_{1}, g_{2}\right)=\rho_{1}\left(g_{1} A, g_{2} A\right)$ and $\rho_{H}\left(h_{1}, h_{2}\right)=\rho_{2}\left(h_{1} A, h_{2} A\right)$. Defining $\rho_{X}$ from $\rho_{G}$ and $\rho_{H}$ using equation (1) of [9] we see that $\rho_{X}$ is a metric. Using 
Thompson [23] we then see that $K\left(G \amalg_{A} H\right)$ in the topology $\tau$ has no small subgroups. Also it is shown in [8] that the amalgamated direct product $G \times{ }_{A} H$ has no small subgroups. Let $U$ and $V$ be open neighbourhoods of $e$ in $G \times{ }_{A} H$ and $K\left(G \amalg_{A} H\right)$, respectively, which contain no nontrivial subgroup. Then $U \times V$ is an open neighbourhood of $e$ in $\left(G \times_{A} H\right) \times K\left(G \amalg_{A} H\right)$ with the topology $\tau$, described above. Therefore $U \times V$ is an open neighbourhood of $e$ in $G \amalg_{A} H$. Now let $S$ be any subgroup of $G \amalg_{A} H$ contained in $U \times V$. If $\Lambda: G \amalg_{A} H \rightarrow G \times{ }_{A} H$ is the canonical homomorphism, then $\Lambda(S) \subseteq U$ and hence $\Lambda(S)=\{e\}$. This implies that $S \subseteq\{e\}$ $\times V$ and so $S \subseteq V \subseteq K\left(G \amalg_{A} H\right)$. This implies that $S=\{e\}$, and thus $G \amalg_{A} H$ has no small subgroups.

Open question. If $A$ is a common closed central subgroup of the Hausdorff topological groups $G$ and $H$, where $G$ and $H$ have no small subgroups, is it necessarily true that $G \amalg_{A} H$ has no small subgroups?

Morris and Nickolas [17] prove that if $G$ and $H$ are any nontrivial groups (that is, $G \neq\{e\}$ and $H \neq\{e\})$ then the only locally compact Hausdorff topology on $G * H$ which makes it into a topological group is the discrete topology. Our next result gives a necessary and sufficient condition for a topology on an amalgamated free product $G *_{A} H$ to be a locally compact Hausdorff group topology.

THEOREM 3. Let $A$ be a common normal subgroup of groups $G$ and $H$ with $A \neq G$ and $A \neq H$. Then a group topology $\tau_{1}$ on $G *_{A} H$ is locally compact Hausdorff if and only if $A$ is locally compact Hausdorff and is an open set in $\tau_{1}$.

Proof. If $A$ is locally compact Hausdorff and open, then $\tau_{1}$ is clearly a locally compact Hausdorff topology.

Conversely, if $\tau_{1}$ is locally compact Hausdorff, consider the canonical (not necessarily continuous) homomorphism $\beta:\left(G *_{A} H, \tau_{1}\right) \rightarrow\left(G / A * H / A, \tau_{2}\right)$, where $\tau_{2}$ is the discrete topology. By Theorem 3 of [17] $\beta$ is a continuous map. As $\tau_{2}$ is discrete, the kernel of $\beta$ must be open in $\tau_{1}$. But the kernel of $\beta$ is $A$; that is, $A$ is open in $\tau_{1}$. Then $A$ is an open (and hence closed) subgroup of the locally compact Hausdorff group ( $G *_{A} H, \tau_{1}$ ), and so it is locally compact and Hausdorff.

RemarKs. (i) Since we announced the above result in [9] we have received a preprint [1] from Alperin which shows, using an entirely different method, that the condition "normal" can be removed in the above theorem. (Alperin's proof uses "groups acting on trees".)

(ii) Theorem 3 does not address itself to the real question: Given $A$ a common subgroup of topological groups $G$ and $H$, under what conditions on $A, G$ and $H$ is $G \amalg_{A} H$ locally compact Hausdorff? We address ourselves to this question now.

Firstly we prove an important result.

THEOREM 4. Let $A$ be a common central subgroup of topological groups $G$ and $H$. Then $G \amalg_{A} H$ is homeomorphic to $\left(G \times{ }_{A} H\right) \times K\left(G \amalg_{A} H\right)$, where $G \times{ }_{A} H$ is the amalgamated direct product and $K\left(G \amalg_{A} H\right)$ has the topology induced as a subspace of $G \amalg_{A} H$.

Proof. We have seen in Lemma 3 of [9] that each element $u$ of $G \amalg_{A} H$ has a unique representation $u=y k$, where $k \in K\left(G \amalg_{A} H\right)$ and $y=g h, g \in G, h \in H$. 
From this we see that there is a natural bijection

$$
\xi: G \amalg_{A} H \rightarrow\left(G \times_{A} H\right) \times K\left(G \amalg_{A} H\right) .
$$

( $\xi$ is, in general, not a homomorphism.)

Consider the diagram

$$
G \amalg H \quad \stackrel{\Psi}{\rightarrow} G \amalg_{A} H \stackrel{\Lambda}{\rightarrow} G \times_{A} H
$$

where $\Psi$ and $\Lambda$ are the canonical continuous homomorphisms. If we denote the subspace $\{g h: g \in G, h \in H\}$ of $G \amalg H$ by $T$ and the subspace $\{g h: g \in G, h \in H\}$ of $G \amalg_{A} H$ by $P$, then we obtain the diagram

$$
T \stackrel{\Psi}{\rightarrow} P \stackrel{\Lambda}{\rightarrow} G \times \times_{A} H .
$$

It is shown in [8] that the map $\Lambda \Psi$ is a quotient map. Thus $\Lambda$ is a homeomorphism. But as $G \amalg_{A} H$ is a topological group, the product map $P \times K\left(G \amalg_{A} H\right) \rightarrow G \amalg_{A} H$, which takes $(y, k) \rightarrow y k$, is continuous. Thus $\xi^{-1}:\left(G \times{ }_{A} H\right) \times K\left(G \amalg_{A} H\right) \rightarrow$ $G \amalg_{A} H$ is continuous.

Observing that $\xi$ is the composition of the continuous maps

(i)

$$
\begin{array}{ccccccc}
G \amalg_{A} H & \rightarrow & \left(G \times_{A} H\right) & \times & \left(G \times{ }_{A} H\right) & \times & \left(G \amalg_{A} H\right) \\
y k & \rightarrow & (y & , & y & , & y k),
\end{array}
$$

$$
\begin{array}{ccccccccccc}
\left(G \times \times_{A} H\right) & \times & \left(G \times{ }_{A} H\right) & \times & \left(G \amalg_{A} H\right) & \rightarrow & \left(G \times{ }_{A} H\right) & \times & \left(G \times_{A} H\right) & \times & \left(G \amalg_{A} H\right) \\
(y & , & y & , & y k) & \rightarrow & (y & , & y^{-1} & , & y k),
\end{array}
$$

$$
\begin{array}{cccccccccccc}
\left(G \times \times_{A} H\right) & \times & \left(G \times_{A} H\right) & \times & \left(G \amalg_{A} H\right) & \rightarrow & \left(G \times{ }_{A} H\right) & \times & \left(G \amalg_{A} H\right) & \times & \left(G \amalg_{A} H\right) \\
(y & , & y^{-1} & , & y k) & \rightarrow & (y & , & y^{-1} & , & y k),
\end{array}
$$

$$
\begin{array}{cccccc}
\left(G \times_{A} H\right) & \times & \left(G \amalg_{A} H\right) & \times & \left(G \amalg_{A} H\right) \\
(y & , & y^{-1} & , & y k) & \\
& & \rightarrow & \left(G \times_{A} H\right) \times\left(G \amalg_{A} H\right), \\
& & \rightarrow & \left(y, y^{-1}(y k)\right)=(y, k) \in\left(G \times_{A} H\right) \times K\left(G \amalg_{A} H\right),
\end{array}
$$

we obtain that $\xi$ is also continuous, and so is a homeomorphism.

Remarks. (i) We note that it follows from the definition of $G \amalg_{A} H$ that the map $\Psi$ is a quotient homomorphism.

(ii) Under the conditions of Theorem 4, if $A$ is closed in $G$ and $H$ then $G$ and $H$ are closed subgroups of $G \amalg_{A} H$, since they are closed subgroups of $G \times{ }_{A} H$.

PROPOSITION 4. Let A be a common open central subgroup of Hausdorff topological groups $G$ and $H$. Then $G \amalg_{A} H$ is homeomorphic to $\left(G \times_{A} H\right) \times D$, where $D$ is a discrete free group. 
Proof. By Theorem 4, $G \amalg_{A} H$ is homeomorphic to $\left(G \times_{A} H\right) \times K\left(G \amalg_{A} H\right)$ where $K\left(G \amalg_{A} H\right)$ is given the subspace topology. Clearly this topology on $K\left(G \amalg_{A} H\right)$, is finer than the topology $\tau$, where $\tau$ is the topology constructed in [9].

It is readily deduced from equation (1) of [9] that the family of all $\rho_{X}$ so defined describes a discrete topology on $X$, as $A$ is open in $G$ and $H$. Further, the extensions of these $\rho_{X}$ to $K\left(G \amalg_{A} H\right)$, as described in Step 2 of [9], yields a discrete topology on $K\left(G \amalg_{A} H\right)$; that is, $\tau$ induces a discrete topology on $K\left(G \amalg_{A} H\right)$. Hence the topology of $G \amalg_{A} H$, being finer than $\tau$, also induces the discrete topology on $K\left(G \amalg_{A} H\right)$. Finally, recall that Proposition 1 of [9] says that $K\left(G \amalg_{A} H\right)$ is a free group.

REMARK. It is interesting to note that under the conditions of Proposition 4, $G *_{A} H$ with the topology $\tau$ is actually $G \amalg_{A} H$.

Open question. Let $A$ be a common closed central subgroup of Hausdorff topological groups $G$ and $H$. What conditions on $A, G$ and $H$ are necessary and sufficient to guarantee that $G *_{A} H$ with the topology $\tau$ is $G \amalg_{A} H$ ?

COROLlaRY 1. Let A be a common open central subgroup of Hausdorff topological groups $G$ and $H$. Then $G \amalg_{A} H$ is locally isomorphic to $G \times_{A} H$.

Proof. Simply observe that the quotient group $G \amalg_{A} H / K\left(G \amalg_{A} H\right)$ is topologically isomorphic to $G \times_{A} H$ and that, by Proposition 4, $K\left(G \amalg_{A} H\right)$ is discrete.

COROLlaRY 2. Let $A$ be a common open central subgroup of topological groups $G$ and $H$. Then $G \amalg_{A} H$ is a Lie group if and only if $G$ and $H$ are Lie groups.

Proof. That $G \amalg_{A} H$ is a Lie group follows from Corollary 1 and the observation in [8] that an amalgamated direct product of Lie groups is a Lie group. Conversely, if $G \amalg_{A} H$ is a Lie group, then so are its closed subgroups $G$ and $H$.

COROllary 3. Let A be a common central subgroup of Hausdorff topological groups $G$ and $H$. Then $A$ is an open subgroup of $G \amalg_{A} H$ if and only if $A$ is an open subgroup of both $G$ and $H$.

Proof. The only part requiring proof is that if $A$ is open in $G$ and $H$ then $A$ is open in $G \times{ }_{A} H$ (and hence by Proposition 4, in $G \amalg_{A} H$ ). But $A \times A$ is open in $G \times H$ and so its image, namely $A$, is open in the quotient group $G \times{ }_{A} H$.

Remark. Observe that if $A$ is an open subgroup of $G \amalg_{A} H$ then $G$ and $H$ (which contain $A$ ) are also open subgroups of $G \amalg_{A} H$. Conversely, if $G$ and $H$ are open in $G \amalg_{A} H$, then $A=H \cap G$ is open in $G \amalg_{A} H$ too.

THEOREM 5. Let $A$ be a common central subgroup of Hausdorff topological groups $G$ and $H$ with $A \neq G$ and $A \neq H$. Then $G \amalg_{A} H$ is locally compact Hausdorff if and only if $A$ is locally compact Hausdorff and is an open subgroup of both $G$ and $H$.

Proof. If $G \amalg_{A} H$ is locally compact Hausdorff then Theorem 3 says that $A$ is locally compact Hausdorff and is an open subgroup of $G \amalg_{A} H$. Therefore $A$ is open in $G$ and $H$.

Conversely, assume that $A$ is open in $G$ and $H$. Then Proposition 4 says that $G \amalg_{A} H$ is homeomorphic to $\left(G \times{ }_{A} H\right) \times D$, where $D$ is discrete. The proof is 
completed by noting that an amalgamated direct product of locally compact groups is locally compact [8].

COROLlaRY 1. Let $A$ be a common central subgroup of topological groups $G$ and $H$ with $A \neq G$ and $A \neq H$. If $G \amalg_{A} H$ is locally compact Hausdorff, then it is homeomorphic to $\left(G \times{ }_{A} H\right) \times D$, where $D$ is a discrete free group.

CoRollary 2. Let $A$ be a common central subgroup of topological groups $G$ and $H$ with $A \neq G$ and $A \neq H$. Then $G \amalg_{A} H$ is not a compact Hausdorff group.

Recall that a topological space $Y$ is said to be a Baire space if for every closed countable covering $\left\{Y_{n}: n=1,2, \ldots\right\}$ of $Y$ at least one of the $Y_{n}$ contains an open set; that is, the interior of $Y_{n} \neq \varnothing$. The most important examples of Baire spaces are locally compact Hausdorff spaces and complete metric spaces. We now proceed to extend Theorem 5 to Baire spaces.

First we record a result of Sidney A. Morris and Edward T. Ordman which appears in their (as yet) unpublished monograph on free topological groups and free products of topological groups.

TheOREM. If $G$ and $H$ are topological groups with $G \neq\{e\}$ and $H \neq\{e\}$, then $G \amalg H$ is a Baire space if and only if $G, H$ and $G \amalg H$ are discrete.

Proof. If $G \amalg H$ is discrete, then it is a Baire space. Conversely, if $G \amalg H$ is a Baire space, then as $G \amalg H$ is homeomorphic to $G \times H \times K(G \amalg H)$, we see that $K(G \amalg H)$, being an open continuous image of a Baire space, is a Baire space. A routine argument shows that if $K(G \amalg H)$ is discrete, then $G, H$ and $G \amalg H$ are discrete. To see that $K(G \amalg H)$ is discrete, observe that as $K(G \amalg H)$ has a topology finer than that in Graev's construction [5], each of the sets $\operatorname{gp}_{n}(K(G \amalg H))$ is a closed subset of $K(G \amalg H)$, where

$$
\begin{aligned}
\operatorname{gp}_{n}(K(G \amalg H))=\left\{\left[g_{1}, h_{1}\right]^{\varepsilon_{1}} \cdots\left[g_{m}, h_{m}\right]^{\varepsilon_{m}}: \varepsilon_{i} \in \mathbf{Z},\right. \\
\left.\sum_{i=1}^{m}\left|\varepsilon_{i}\right| \leqslant n, g_{i} \in G \text { and } h_{i} \in H, i=1, \ldots, m\right\} .
\end{aligned}
$$

As $K(G \amalg H)$ is a Baire space and $K(G \amalg H)=\cup_{n=1}^{\infty} \mathrm{gp}_{n}(K(G \amalg H))$, we see that $\operatorname{gp}_{n}(K(G \amalg H))$ contains an open subset $U$ of $K(G \amalg H)$, for some $n$. So, by translating $U$, we obtain an open neighbourhood $N$ of $e$ which is wholly contained in $\operatorname{gp}_{k}(K(G \amalg H))$, for some integer $k$. But for any integer $l$, there exists a neighbourhood $N_{l}$ of $e$ such that $\left(N_{l}\right)^{l} \subseteq N$. But it is easily verified (cf. Thompson [23]) that unless $N_{l}=\{e\},\left(N_{l}\right)^{l}$ contains words of reduced length $l$ with respect to the free basis $[G, H] \backslash\{e\}$ of $K(G \amalg H)$. Thus $\left(N_{l}\right)^{l} \nsubseteq N$, for sufficiently large $l$, unless $N_{l}=\{e\}$, as $N \subseteq \mathrm{gp}_{k}(K(G \amalg H))$. Thus $N_{l}=\{e\}$ and $K(G \amalg H)$ is discrete, as required. This completes the proof of Morris and Ordman's theorem.

Now we can prove the result we want. 
THEOREM 6. Let $A$ be a common central subgroup of topological groups $G$ and $H$ with $A \neq G$ and $A \neq H$. Then $G \amalg_{A} H$ is a Baire space if and only if $A$ is an open subgroup of $G$ and $H$ and $G \times{ }_{A} H$ is a Baire space.

Proof. If $G \amalg_{A} H$ is a Baire space then so is its quotient group $G / A \amalg H / A$. But by the Morris-Ordman theorem above, this implies that $G / A$ and $H / A$ are discrete. So $A$ is open in $G$ and $H$. As $G \times{ }_{A} H$ is an open continuous image of $G \amalg_{A} H$, it is a Baire space.

Conversely, if $A$ is open in $G$ and $H$, then $G \amalg_{A} H$ is homeomorphic to $\left(G \times{ }_{A} H\right)$ $\times D$, where $D$ is discrete. So if $G \times{ }_{A} H$ is a Baire space, then so too is $G \amalg_{A} H$.

REMARK. It is clear that if $G$ and $H$ are Baire spaces then so is $G \times{ }_{A} H$. However, the converse is false. For example, if $\mathbf{Q}_{i} \subset \mathbf{R}_{i}, i=1,2$, denote the groups of rational numbers and real numbers with their usual topologies, then

$$
\left(\mathbf{R}_{1} \times \mathbf{Q}_{2}\right) \times \mathbf{Q}_{1} \times \mathbf{Q}_{2}\left(\mathbf{Q}_{1} \times \mathbf{R}_{2}\right)
$$

is a Baire space as it is topologically isomorphic to $\mathbf{R}_{1} \times \mathbf{R}_{2}$, however, neither $\mathbf{R}_{1} \times \mathbf{Q}_{2}$ nor $\mathbf{Q}_{1} \times \mathbf{R}_{2}$ is a Baire space.

However, we do not know the answer to the

Open question. Let $A$ be a common open central subgroup of topological groups $G$ and $H$. If $G \times{ }_{A} H$ is a Baire space, are $G$ and $H$ necessarily Baire spaces?

In the special case of complete metric groups we can obtain the desired result.

Firstly we state an obvious result.

Proposition 5. Let $A$ be a common closed central subgroup of topological groups $G$ and $H$. Then $G \times{ }_{A} H$ is a complete metric group if and only if $G$ and $H$ are complete metric groups.

THEOREM 7. Let $A$ be a common central subgroup of topological groups $G$ and $H$ with $A \neq G$ and $A \neq H$. Then $G \amalg_{A} H$ is a complete metric group if and only if $A$ is an open subgroup of $G$ and $H$, and $G$ and $H$ are complete metric groups.

Proof. The same argument as in Theorem 6 shows that $G \amalg_{A} H$ is a complete metric group if and only if $G \times{ }_{A} H$ is a complete metric group and $A$ is open in $G$ and $H$. The result then follows from Proposition 5 .

COROLlaRY. If $A$ is a common central subgroup of topological groups $G$ and $H$ such that $G \amalg_{A} H$ is a complete metric group, then $G \amalg_{A} H$ is homeomorphic to $\left(G \times{ }_{A} H\right) \times$ $D$, where $D$ is a discrete free group.

We now turn to the consideration of maximally almost periodic (MAP-) groups. Recall that a topological group is said to be $M A P$ if it admits a continuous one-to-one homomorphism into a compact Hausdorff group. For example, every locally compact abelian group and every compact Hausdorff group is a MAP-group.

Using the main result of Hulanicki [6], Morris [13] showed that if $G$ and $H$ are MAP-groups then $G \amalg H$ is a MAP-group. \{The reader's attention is drawn to the corrigendum [13]. The error pointed out there is carried over from Hulanicki [6], however the proofs of the relevant Theorem 2.11 of Morris [13] and the main theorem of Hulanicki are correct. 
The following lemma is probably well known and its proof is obvious.

LEMMA 2. Let $K$ be a compact normal subgroup of a MAP-group $G$. Then $G / K$ is a MAP-group.

PROPOSITION 6. Let $A$ be a common compact central subgroup of MAP-groups $G$ and $H$. Then the amalgamated direct product $G \times{ }_{A} H$ is a MAP-group.

Proof. If $G$ and $H$ are MAP-groups, then clearly $G \times H$ is a MAP-group. Then $G \times{ }_{A} H$ being a quotient group of the MAP-group $G \times H$ by a compact Hausdorff central subgroup is, by Lemma 1, a MAP-group.

THEOREM 8. Let $A$ be a common compact central subgroup of topological groups $G$ and $H$. Then $G \amalg_{A} H$ is a MAP-group if and only if $G$ and $H$ are MAP-groups.

Proof. If $G \amalg_{A} H$ is a MAP-group then clearly its subgroups $G$ and $H$ are MAP-groups.

Conversely, let $x \neq e$ be any element of $G \amalg_{A} H$. It suffices to show that there is a continuous homomorphism of $x$ into a compact Hausdorff group which does not map $x$ to the identity element. Indeed, it is enough to show that there exists a continuous homomorphism of $G$ into a MAP-group which does not map $x$ to the identity element. If $x \notin K\left(G \amalg_{A} H\right)$ then the canonical homomorphism of $G \amalg_{A} H$ into the MAP-group $G \times{ }_{A} H$ is such a map. If $x \in K\left(G \amalg_{A} H\right)$ then Proposition 1 of [9], shows that the canonical homomorphism $\beta$ of $G \amalg_{A} H$ onto $G / A \amalg H / A$ is the required map, because $G / A \amalg H / A$ being the free product of MAP-groups is, as noted above, a MAP-group.

Closely related to the concept of MAP-group is that of locally invariant (or SIN-) group. A topological group is said to be locally invariant if every neighbourhood of the identity contains a neighbourhood invariant under all inner automorphisms. Every abelian topological group and every compact Hausdorff group is locally invariant. Within the class of connected locally compact Hausdorff abelian groups the properties "locally invariant" and "maximally almost periodic" coincide. However, there exist locally compact groups which are MAP-groups but not locally invariant and vice-versa.

We have seen that free products of MAP-groups are MAP-groups. However, the situation is quite different for locally invariant groups. Indeed, Morris [15] proved that if $G$ and $H$ are connected locally compact (locally invariant) Hausdorff groups with $G \neq\{e\}$ and $H \neq\{e\}$, then $G \amalg H$ is not locally invariant. Thus the following positive result is of interest.

TheOREM 9. Let $A$ be a common open central subgroup of Hausdorff topological groups $G$ and $H$. Then $G \amalg_{A} H$ is a locally invariant group.

Proof. As $A$ is open in $G$ and $H$, Corollary 3 of Proposition 4 implies that $A$ is open in $G \amalg_{A} H$. From this and the centrality of $A$, it immediately follows that $G \amalg_{A} H$ is locally invariant. 
A topological group is said to be almost locally invariant if it admits a continuous one-to-one homomorphism into a locally invariant group. Of course, locally invariant groups are almost locally invariant but the converse is false [21]. However, every connected locally compact almost locally invariant group is locally invariant [21].

Ordman and Morris observed that free products of almost locally invariant groups are almost locally invariant. Noticing that they also have a result (Proposition 2) which is the analogue of our Lemma 2 for almost locally invariant groups we can use an argument exactly like that in Theorem 8 to obtain

THEOREM 10. Let $A$ be a common compact central subgroup of Hausdorff topological groups $G$ and $H$. Then $G \amalg_{A} H$ is almost locally invariant if and only if $G$ and $H$ are almost locally invariant.

Open questions. (1) If $A$ is a common closed central subgroup of MÄP-groups $G$ and $H$, is $G \amalg_{A} H$ necessarily a MAP-group?

(2) If $A$ is a common closed central subgroup of almost locally invariant Hausdorff groups $G$ and $H$, are $G \times_{A} H$ and $G \amalg_{A} H$ necessarily almost locally invariant groups?

4. $k_{\omega}$-groups. For general topological groups we have proved (Theorem 4 ) that $G \amalg_{A} H$ is homeomorphic to $\left(G \times_{A} H\right) \times K\left(G \amalg_{A} H\right)$, where $K\left(G \amalg_{A} H\right)$ is topologized as a subspace of $G \amalg_{A} H$. However, we do not know exactly what the topology of $K\left(G \amalg_{A} H\right)$ is. The best we can say is that it is finer than that used in the construction presented in [9]. However, for $k_{\omega}$-groups we are able to satisfactorily describe the topology of $K\left(G \amalg_{A} H\right)$, and hence also of $G \amalg_{A} H$. \{A Hausdorff space $X$ is said to be a $k_{\omega}$-space [18] with $k_{\omega}$-decomposition $X=\cup X_{n}$, if $X_{1}, X_{2}, \ldots$ are compact subsets of $X, X_{n} \subseteq X_{n+1}$ for all $n$, and the $X_{n}$ 's determine the topology of $X$ in the sense that a subset $A$ of $X$ is closed if and only if $A \cap X_{n}$ is compact, for all n.

THEOREM 11. Let $A$ be a common closed central subgroup of $k_{\omega}$-groups $G$ and $H$. Then the cartesian subgroup $K\left(G \amalg_{A} H\right)$ of $G \amalg_{A} H$ is the Graev free topological group $F(G / A \wedge H / A)$ on the smash product of $G / A$ and $H / A$.

Proof. [Recall that the smash product [2] of $G / A$ and $H / A$ is the quotient space of $G / A \times H / A$ obtained by identifying the set $(\{e\} \times H / A) \cup(G / A \times\{e\})$ with $\{e\}$.

Theorem 4 of [18] says that in $G \amalg H$, the set $[G, H]=\left\{g^{-1} h^{-1} g h: g \in G, h \in H\right\}$ has the smash product topology $G \wedge H$, while in $G / A \amalg H / A$ the set $[G / A, H / A]$ has the topology of $G / A \wedge H / A$. Thus the canonical homomorphism $\Delta: G \amalg H \rightarrow$ $G / A \amalg H / A$, when restricted to $[G, H]$, is a quotient map of $[G, H]$ onto $[G / A, H / A]$. It is readily verified (and well known) that if $X$ and $Y$ are completely regular spaces and $\phi$ is a quotient mapping of $X$ onto $Y$, then the canonical homomorphism of the free topological group on $X$ onto the free topological group on $Y$ which extends $\phi$ is also a quotient map. Thus we obtain a quotient homomorphism of $F(G \wedge H)$ onto $F(G / A \wedge H / A)$. But Theorem 5 of [18] says that $K(G \amalg H)$ 
is $F(G \wedge H)$ and $K(G / A \amalg H / A)$ is $F(G / A \wedge H / A)$. So $\Delta$, restricted to $K(G \amalg H)$, is a quotient homomorphism of $K(G \amalg H)$ onto $K(G / A \amalg H / A)$. Now consider the diagram

$$
K(G \amalg H) \quad \stackrel{\Psi}{\rightarrow} K\left(G \amalg_{A} H\right) \quad \stackrel{\Lambda}{\rightarrow} \quad K(G / A \amalg H / A) .
$$

Observing that $\Psi$ and $\Lambda$ are surjective continuous homomorphisms, that $\Lambda$ is injective and that $\Lambda \Psi=\Delta$ is a quotient homomorphism we see that $\Lambda$ is a topological group isomorphism of $K\left(G \amalg_{A} H\right)$ onto $K(G / A \amalg H / A)$. Thus $K\left(G \amalg_{A} H\right)$ is $F(G / A \wedge H / A)$.

As an immediate corollary of Theorems 4 and 11 and the fact that a finite product of $k_{\omega}$-spaces is a $k_{\omega}$-space [20] we obtain

THEOREM 12. Let $A$ be a common closed central subgroup of $k_{\omega}$-groups $G$ and $H$. Then $G \amalg_{A} H$ is a $k_{\omega}$-group and is homeomorphic to $\left(G \times{ }_{A} H\right) \times F(G / A \wedge H / A)$, where $F(G / A \wedge H / A)$ is the Graev free topological group on the smash product of $G / A$ and $H / A$.

REMARKS. In [10] it is shown that if the Hausdorff group $G$ is algebraically generated by the $k_{\omega}$-space $X=\cup X_{n}$ and $G$ has the finest group topology which will induce the given topology on $X$, then $G$ is a $k_{\omega}$-space with $k_{\omega}$-decomposition $G=\cup \operatorname{gp}_{n}\left(X_{n}\right)$, where $\operatorname{gp}_{n}\left(X_{n}\right)$ denotes the set $\left(X_{n} \cup\left(X_{n}\right)^{-1} \cup\{e\}\right)^{n}$. In particular if $X=\cup X_{n}$ is a $k_{\omega}$-space, then the free topological group on $X$ has $k_{\omega}$-decomposition $F(X)=\cup \mathrm{gp}_{n}\left(X_{n}\right)$. Thus we can use Theorem 12 to write down a $k_{\omega}$-decomposition of $G \amalg_{A} H$.

Note that a different $k_{\omega}$-decomposition can also be written down easily. If $G$ and $H$ have $k_{\omega}$-decompositions $G=\cup G_{n}$ and $H=\cup H_{n}$, respectively, then $G \amalg_{A} H$ has $k_{\omega}$-decomposition $G \amalg_{A} H=\cup \mathrm{gp}_{n}\left(G_{n} \cup H_{n}\right)$. [This is so since $G \amalg_{A} H$ has the finest group topology which will induce the given topology on the subspace $G \cup H$ of $G \amalg_{A} H$.]

In [18] it was proved that if $G_{i}, H_{i}, i=1,2$, the $k_{\omega}$-groups with $G_{1}$ homeomorphic to $G_{2}$ and $H_{1}$ homeomorphic to $H_{2}$, then $G_{1} \amalg H_{1}$ is homeomorphic to $G_{2} \amalg H_{2}$. We now record a result for amalgamated free products which includes this as a special case.

As an immediate consequence of Theorem 12 we have

THEOREM 13. Let $A_{i}$ be a common closed central subgroup of the $k_{\omega}$-groups $G_{i}$ and $H_{i}$, for $i=1,2$. If

(a) $G_{1} \times{ }_{A_{1}} H_{1}$ is homeomorphic to $G_{2} \times{ }_{A_{2}} H_{2}$, and

(b) $G_{1} / A_{1} \wedge H_{1} / A_{1}$ is homeomorphic to $G_{2} / A_{2} \wedge H_{2} / A_{2}$, then $G_{1} \amalg_{A_{1}} H_{1}$ is homeomorphic to $G_{2} \amalg_{A_{2}} H_{2}$.

COROLlARY 1. If $G_{i}, H_{i}$ are $k_{\omega}$-groups for $i=1,2$ such that

(i) $G_{1} \times H_{1}$ is homeomorphic to $G_{2} \times H_{2}$, and

(ii) $G_{1} \wedge H_{1}$ is homeomorphic to $G_{2} \wedge H_{2}$, then $G_{1} \amalg H_{1}$ is homeomorphic to $G_{2} \amalg H_{2}$. 
COROllaRY 2. If $G_{i}, H_{i}$ are $k_{\omega}$-groups for $i=1,2$ with $G_{1}$ homeomorphic to $G_{2}$ and $H_{1}$ homeomorphic to $H_{2}$, then $G_{1} \amalg H_{1}$ is homeomorphic to $G_{2} \amalg H_{2}$.

In $\S 3$ we were unable to find necessary and sufficient condtions on $A, G$ and $H$ for $G \amalg_{A} H$ to be a Baire space. For the $k_{\omega}$-case we can find such conditions. Observe firstly that a $k_{\omega}$-group is a Baire space if and only if it is locally compact [16]. So from Theorem 5 and Theorem 12 we deduce

THEOREM 14. Let $A$ be a common closed central subgroup of $k_{\omega}$-groups $G$ and $H$, with $A \neq G$ and $A \neq H$. Then $G \amalg_{A} H$ is a Baire space if and only if $A$ is an open subgroup of both $G$ and $H$, and $A$ is a locally compact group.

In $\S 3$ we settled the question of when $G \amalg_{A} H$ is a complete metric group. For $k_{\omega}$-groups we can decide when $G \amalg_{A} H$ is a metric group. The key observation is that a metric $k_{\omega}$-space is locally compact [3]. Consequently, from Theorems 12 and 5 we can deduce

THEOREM 15. Let $A$ be a common closed central subgroup of $k_{\omega}$-groups $G$ and $H$, with $A \neq G$ and $A \neq H$. Then $G \mathrm{\amalg}_{A} H$ is a metric group if and only if $A$ is an open subgroup of both $G$ and $H$ and $A$ is a metric group.

For a moment we digress from our consideration of $k_{\omega}$-groups and prove a theorem for more general groups which we shall then strengthen in the case of $k_{\omega}$-groups.

THEOREM 16. Let $A$ be a common closed central subgroup of Hausdorff groups $G$ and $H$. If $G_{1}$ is a closed subgroup of $G, H_{1}$ is a closed subgroup of $H, G_{1} \supseteq A$, and $H_{1} \supseteq A$, then

(i) the subgroup $D=\operatorname{gp}\left(G_{1} \cup H_{1}\right)$ of $G \times{ }_{A} H$ is closed in $G \times{ }_{A} H$ and is topologically isomorphic to $G_{1} \times{ }_{A} H_{1}$, and

(ii) the subgroup $E=\operatorname{gp}\left(G_{1} \cup H_{1}\right)$ of $G \amalg_{A} H$ is closed in $G \amalg_{A} H$.

Proof. The natural map $\gamma: G \times H \rightarrow G \times{ }_{A} H$ is a quotient map [8] and as $G_{1}$ and $H_{1}$ both contain $A$, we see that $\gamma^{-1}\left(\operatorname{gp}\left(G_{1} \cup H_{1}\right)\right)=G_{1} \times H_{1}$, which is closed in $G \times H$. Thus $D=\operatorname{gp}\left(G_{1} \cup H_{1}\right)$ is closed in $G \times{ }_{A} H$. Further, the kernel $\left\{\left(a, a^{-1}\right)\right.$ : $a \in A\}$ of the map $\gamma$ is contained in $G_{1} \times H_{1}$. Thus $\gamma$ restricted to $G_{1} \times H_{1}$ is an open map of $G_{1} \times H_{1}$ onto $D$ and has kernel $\left\{\left(a, a^{-1}\right) ; a \in A\right\}$. Thus $D$ is topologically isomorphic to $G_{1} \times{ }_{A} H_{1}$.

It is clear that the group $E$ is algebraically the amalgamated free product $G_{1} *_{A} H_{1}$. Note that $G \amalg_{A} H$ is homeomorphic to $\left(G \times_{A} H\right) \times K\left(G \amalg_{A} H\right)$, where $K\left(G \amalg_{A} H\right)$ has the subspace topology, and observe that under this homeomorphism $D$ maps to $\left(G_{1} \times{ }_{A} H_{1}\right) \times V$, where $V$ is the subgroup of $K\left(G \amalg_{A} H\right)$ generated by $\left[G_{1}, H_{1}\right]$. The previous paragraph tells us that $G_{1} \times{ }_{A} H_{1}$ is closed in $G \times{ }_{A} H$, so to prove that $E$ is closed we only have to show that $V$ is closed in $K\left(G \amalg_{A} H\right)$.

Of course we do not know what the topology on $K\left(G \amalg_{A} H\right)$ is, but we do know that it is finer than the topology $\tau$ described in [9]. Thus to prove that $E$ is closed, it suffices to show that $V$ is closed in the topology $\tau$. Using an argument similar to that of Lemma 1 we can show that $\left[G_{1}, H_{1}\right]$ is a closed subset of $[G, H] \subset K\left(G \amalg_{A} H\right)$ in the topology $\tau$. Then by Graev [4, §4D], it follows that $V=\operatorname{gp}\left[G_{1}, H_{1}\right]$ is closed in $\tau$. 
Remarks. (i) Theorem 16(ii) says less than we would like it to say. It would be nice if $E$ were, in fact, topologically isomorphic to $G_{1} \amalg_{A} H_{1}$-but this is probably too much to hope for in general. However, it is true for $k_{\omega}$-groups. We see this in Theorem 17.

(ii) If the conditions that $G_{1} \supseteq A$ and $H_{1} \supseteq A$ were dropped in Theorem 16, it is plausible that the subgroup $\operatorname{gp}\left(G_{1} \cup H_{1}\right)$ of $G \times{ }_{A} H$ would be topologically isomorphic to $G_{1} \times_{A_{1}} H_{1}$, where $A_{1}=G_{1} \cap H_{1} \subseteq G \times{ }_{A} H$. However, the following example shows that this is not the case.

ExAmple. Let $G=\mathbf{R}, H=\mathbf{R}$ and $A=\alpha \mathbf{Z}=\operatorname{gp}(\{\alpha\}) \subseteq \mathbf{R}$, where $\alpha$ is an irrational number. Let $G_{1}=\mathbf{Z} \subseteq G$ and $H_{1}=\mathbf{Z} \subseteq H$. Then $G \times H$ is topologically isomorphic to $\mathbf{R} \times \mathbf{R}$, and $G \times_{A} H$ is topologically isomorphic to $\mathbf{R} \times \mathbf{T}$, where $\mathbf{T}$ is the circle group. As $A \cap G_{1}=\{e\}$ and $A \cap H_{1}=\{e\}$, we might expect that the subgroup $\operatorname{gp}\left(G_{1} \cup H_{1}\right)$ of $G \times{ }_{A} H$ would be $G_{1} \times H_{1}$ (that is, $G_{1} \times{ }_{A_{1}} H_{1}$, where $\left.A_{1}=G_{1} \cap H_{1}=\{e\}\right)$, but $G_{1} \times H_{1}=\mathbf{Z} \times \mathbf{Z}$ and $\mathbf{R} \times \mathbf{T}$ does not contain any subgroup topologically isomorphic to $\mathbf{Z} \times \mathbf{Z}$.

THEOREM 17. Let $A$ be a common closed central subgroup of $k_{\omega}$-groups $G$ and $H$. If $G_{1}$ is a closed subgroup of $G$, and $H_{1}$ is a closed subgroup of $H, G_{1} \supseteq A$ and $H_{1} \supseteq A$, then the subgroup $E=\operatorname{gp}\left(G_{1} \cup H_{1}\right)$ of $G \amalg_{A} H$ is topologically isomorphic to $G_{1} \amalg_{A} H_{1}$.

Proof. We have already noted, in Theorem 16, that $E$ is algebraically the amalgamated free product $G_{1} *_{A} H_{1}$, and that it is homeomorphic to $\left(G_{1} \times{ }_{A} H_{1}\right) \times V$, where $V$ is the subgroup of $K\left(G \amalg_{A} H\right)$ generated by $\left[G_{1}, H_{1}\right]$. So in view of Theorems 11 and 12 it only remains to show that $V$ is $F\left(G_{1} / A \wedge H_{1} / A\right)$. Recalling that $K\left(G \amalg_{A} H\right)$ is $F(G / A \wedge H / A)$, (by Theorem 11) and that $V$ is generated by the closed subspace $\left[G_{1}, H_{1}\right]$ of $[G, H]$, Theorem 3 of [10] implies that $V$ is the (Graev) free topological group on $\left[G_{1}, H_{1}\right]$. Here $\left[G_{1}, H_{1}\right]$ has the induced topology from $G / A \wedge H / A$ which is readily seen to be $G_{1} / A \wedge H_{1} / A$. So $V$ is indeed $F\left(G_{1} / A \wedge H_{1} / A\right)$.

The proof of our final result is quite routine and so is omitted.

THEOREM 18. Let $A, B$ and $C$ be any topological groups. Then the amalgamated free product $(A \times B) \amalg_{A}(A \times C)$ exists and is topologically isomorphic to $A \times(B \amalg C)$. If $A, B$ and $C$ are Hausdorff then $(A \times B) \amalg_{A}(A \times C)$ is Hausdorff. If $A, B$ and $C$ are $k_{\omega}$-groups, then $(A \times B) \amalg_{A}(A \times C)$ is homeomorphic to $A \times B \times C \times F(B \wedge C)$.

\section{REFERENCES}

1. R. Alperin, Locally compact groups acting on trees (preprint).

2. R. Brown, Elements of modern topology, McGraw-Hill, London, 1968.

3. S. P. Franklin and B. V. S. Thomas, $A$ survey of $k_{\omega}$-spaces, Topology Proc. 2 (1977), 111-124.

4. M. I. Graev, Free topological groups, Izv. Akad. Nauk SSSR Ser. Mat. 12 (1948), 279-324 (Russian); English transl., Amer. Math. Soc. Transl. 35 (1951). Reprinted in Amer. Math. Soc. Transl. (1) 8 (1962), $305-364$.

5. , On free products of topological groups, Izv. Akad. Nauk SSSR Ser. Mat. 14 (1950), 343-354. (Russian)

6. A. Hulanicki, Isomorphic embeddings of free products of compact groups, Colloq. Math. 16 (1967), 236-241.

7. I. Kaplansky, Lie algebras and locally compact groups, Univ. of Chicago Press, Chicago, 1971.

8. M. S. Khan and S. A. Morris, Amalgamated direct products of topological groups, Math. Chronicle (to appear). 
9. 273 (1982), 405-416.

10. J. Mack, S. A. Morris and E. T. Ordman, Free topological groups and the projective dimension of $a$ locally compact abeliän group, Proc. Amer. Math. Soc. 40 (1973), 303-308.

11. W. Magnus, A. Karrass and D. Solitar, Combinatorial group theory, Interscience, New York, 1966.

12. A. A. Markov, On free topological groups, Izv. Akad. Nauk. SSSR 9 (1945), 3-64 (Russian); English transl., Amer. Math. Soc. Transl. 30 (1950), 11-88. Reprinted in Amer. Math. Soc. Transl. (1) 8 (1962), 195-272.

13. S. A. Morris, Free products of topological groups, Bull. Austral. Math. Soc. 4 (1971), 17-29. Corrigendum, Bull. Austral. Math. Soc. 12 (1975), 480.

14. Varieties of topological groups and left adjoint functors, J. Austral. Math. Soc. 16 (1973), $220-227$.

15. 35 (1976), 21-27.

Local compactness and local invariance of free products of topological groups, Colloq. Math.

16. __ Pontryagin duality and the structure of locally compact abelian groups, Cambridge Univ. Press, London and New York, 1977.

17. S. A. Morris and Peter Nickolas, Locally compact group topologies on an algebraic free product of groups, J. Algebra 38 (1976), 393-397.

18. S. A. Morris, E. T. Ordman and H. B. Thompson, The topology of free products of topological groups, Proc. Second Internat. Conf. Theory of Groups (Canberra, 1973), Lecture Notes in Math., vol. 372, Springer-Verlag, Berlin and New York, pp. 504-515.

19. B. H. Neumann, An essay on free products of groups with amalgamations, Philos. Trans. Royal Soc. London Ser. A 246 (1954), 503-554.

20. E. T. Ordman, Free k-groups and free topological groups, General Topology and Appl. 5 (1975), $205-219$.

21. E. T. Ordman and S. A. Morris, Almost locally invariant topological groups, J. London Math. Soc. (2) 9 (1974), 30-34.

22. B. V. S. Thomas, Categories of topological groups, Quaestiones Math. 2 (1977), 355-377.

23. H. B. Thompson, Remarks on free topological groups with no small subgroups, J. Austral. Math. Soc. 18 (1974), 482-484.

Department of Mathematics, La Trobe University, Bundoora, Victoria, 3083, Australia (Current address of S. A. Morris)

Current address (M. S. Khan): Department of Mathematics, Aligarh Muslim University, Aligarh 202001, India 